\title{
Attainability to Solve Fractional Differential Inclusion on the Half Line at Resonance
}

\author{
Ahmed Salem $\mathbb{D}^{1},{ }^{1}$ Faris Alzahrani, ${ }^{1}$ and Aeshah Al-Dosari ${ }^{1,2}$ \\ ${ }^{1}$ Department of Mathematics, Faculty of Science, King Abdulaziz University, P.O. Box 80203, Jeddah 21589, Saudi Arabia \\ ${ }^{2}$ Prince Sattam University, Al-Kharj, Saudi Arabia \\ Correspondence should be addressed to Ahmed Salem; ahmedsalem74@hotmail.com
}

Received 18 September 2019; Revised 5 June 2020; Accepted 26 June 2020; Published 23 July 2020

Academic Editor: Eulalia Martínez

Copyright ( $\odot 2020$ Ahmed Salem et al. This is an open access article distributed under the Creative Commons Attribution License, which permits unrestricted use, distribution, and reproduction in any medium, provided the original work is properly cited.

The presented article is deduced about the positive solutions of the fractional differential inclusion at resonance on the half line. The fractional derivative used is in the sense of Riemann-Liouville and the problem is supplemented by unseparated conditions. The existence results are illustrated in view of Leggett-Williams theorem due to O'Regan and Zima on unbounded domain.

\section{Introduction}

In this paper, we study the possibility of finding a positive solution on unbounded domain with unseparated conditions for the following fractional differential inclusion:

$$
\begin{gathered}
{ }^{R} D^{\alpha}[\kappa(\tau) \nu(\tau)] \in A(\tau, \nu(\tau)), \quad \tau \in[0, \infty), \\
\nu^{(i)}(0)=0, \quad i=0, \ldots, n-2 ; n \in \mathbb{N}, \\
{ }^{R} D^{\alpha-1}[\kappa(0) \nu(0)]=\lim _{\tau \longrightarrow \infty}{ }^{R} D^{\alpha-1}[\kappa(\tau) \nu(\tau)],
\end{gathered}
$$

which is contingent with $A(\tau, \nu(\tau))=q(\tau) B(\tau, \nu(\tau))$ for which $B:[0, \infty) \times \mathbb{R} \longrightarrow \mathbb{R}$ is multivalued map, $n-1<\alpha<n, n \geq 2, \kappa(\tau), q(\tau) \in B C([0, \infty), \kappa(\tau) \geq 1$, and ${ }^{R} D^{\alpha}$ is the symbol of the Riemann-Liouville fractional derivative.

Riemann-Liouville fractional derivative and corresponding integral and many other types of fractional derivatives and integrals with singular and nonsingular kernels are used in fractional calculus as generalizations of the ordinary differentiations and integrations. Thus, many scientific teams are attracted to link their works with fractional calculus, which has been effectively applied in the other scientific fields. As a result, there are some books talking about fractional calculus and some of its applications with fractional equations and inclusions, see [1-3]. In the existence and uniqueness fields, there are many articles concerned with different types of fractional differential equations or inclusions. For fractional differential equations, there are a huge number of contributions (see [4-9] and the references given therein). For fractional differential inclusions, Salem and Al-Dosari [10] have studied the existence results of solution for fractional SturmLiouville inclusion associated with composition with multimaps. Chen and Tang [11] have investigated probability of the positive solution for the fractional equation below on unbounded domain at resonance:

$$
\begin{aligned}
{ }^{R} D^{\alpha} \nu(\tau) & =g(\tau, \nu(\tau)), \quad \tau \in[0, \infty), \\
v^{(i)}(0) & =0, \quad i=0,1,2,3<\alpha<4, \\
{ }^{R} D^{\alpha-1} \nu(0) & =\lim _{\tau \longrightarrow \infty}{ }^{R} D^{\alpha-1} \nu(\tau) .
\end{aligned}
$$

Jiang and Yang [12] have studied the positive solution for the second ordinary boundary value problems:

$$
\begin{aligned}
\nu^{\prime \prime}(\tau)+g(\tau, \nu(\tau)) & =0, \quad \tau \in[0, \infty), \\
\nu(0) & =0 \\
\nu^{\prime}(+\infty) & =\sum_{i=1}^{m-1} \alpha_{i} \nu^{\prime}\left(\zeta_{i}\right),
\end{aligned}
$$

where $\quad \alpha_{i}>0, \sum_{i=1}^{m-1} \alpha_{i}=1,0=\zeta_{1}<, \ldots,<\zeta_{m-1}<\infty \quad$ and $g(t, 0)$ is not always equal to zero. 
On a bounded domain, Chen et al. [13] have explored sufficient conditions to obtain a positive solution for the problem:

$$
\begin{aligned}
{ }^{R} D^{\alpha} \nu(\tau) & \in g(\tau, \nu(\tau)), \quad \tau \in[0,1], \\
v^{(i)}(0) & =0, \quad i=0,1,2,3<\alpha<4, \\
{ }^{R} D^{\alpha-1} \nu(0) & ={ }^{R} D^{\alpha-1} \nu(1) .
\end{aligned}
$$

Furthermore, $\mathrm{Hu}$ [14] has given some results of positive solution at resonance for the boundary value problem formulated as follows:

$$
\begin{aligned}
{ }^{C} D^{\alpha} \nu(\tau) & =g(\tau, \nu(\tau)), \quad \tau \in[0,1], \\
v^{(i)}(0) & =0, \quad i=1,2, \ldots, n-1, \\
\nu(0) & =v(1), \quad n-1<\alpha<n .
\end{aligned}
$$

By reducing nonperturbed boundary value problems at resonance, Wang and Liu [15] have presented their results about positive solution of the problem:

$$
\begin{aligned}
{ }^{R} D^{\alpha} \nu(\tau)+g(\tau, \nu(\tau)) & =0, \quad \tau \in[0,1], \\
\nu(0) & =0, \quad 1<\alpha<2, \\
\nu(1) & =\beta \nu(\zeta), \quad \beta \zeta^{\alpha-1}=1 .
\end{aligned}
$$

About an overall summary of resonance and its applications, Haller [16] has mentioned that "resonances are regions in the phase space of a dynamical system in which the frequencies of some angular variables become nearly commensurate. Such regions have a profound effect on the dynamics of the system, since they are rich sources of highly complex motions. In molecular dynamics, resonances are known to give rise to chaotic patterns, multiple time scales, and apparent irreversibility in the transfer of energy between different oscillatory states of molecules. In engineering structures, interactions among resonant modes are responsible for most complicated dynamical phenomena, which again include energy transfer, multitime-scale behavior, and chaotic motions. It is of great practical importance to understand the common mechanism behind these irregular features, both qualitatively and quantitatively. The circle of further applications ranges from nonlinear optics through celestial and fluid mechanics to electromagnetism."

Chaotic patterns, oscillatory states of molecules, complicated dynamical phenomena, and multitime-scale behavior are all given a strong cause to study differential problems at resonance associated with multivalued maps, which are not presented until now. So, our aim is to show the possibility of having at least one positive solution at resonance. For the sake of that, in Section 2, we give some basic notations and results of fractional differentiations and integrations, some facts of multimaps and their arguments, and some needed lemmas and fixed point theorems. Section 3 contains some required results to the existence of positive solution for the given problem. Before the conclusion, Section 4 shows a quintessence as an application of our outcomes.

\section{Precursory Notations}

This section is devoted to provide some notations and definitions for the fractional calculus, multivalued functions, and Fredholm operator.

2.1. Fractional Calculus. We introduce some definitions and fundamental rules on the fractional calculus that are needed for the new results $[1,17,18]$.

Definition 1. For $\zeta>0$, the Riemann-Liouville fractional integral of a continuous function $h(\tau):[0, \infty) \longrightarrow \mathbb{R}$ is defined by

$$
I^{\zeta} h(\tau)=\frac{1}{\Gamma(\zeta)} \int_{0}^{\tau}(\tau-t)^{\zeta-1} h(t) \mathrm{d} t
$$

Definition 2. For $n \in \mathbb{N}$ and $n-1 \leq \zeta<n$, the Riemann-Liouville fractional derivative of an $n$th absolutely continuous function $h(\tau):[0, \infty) \longrightarrow \mathbb{R}$ is given by

$$
{ }^{R} D^{\zeta} h(\tau)=\frac{1}{\Gamma(n-\zeta)}\left(\frac{\mathrm{d}}{\mathrm{d} \tau}\right)^{n} \int_{0}^{\tau}(\tau-t)^{n-\zeta-1} h(t) \mathrm{d} t .
$$

Lemma 1. Let $h(\tau) \in L^{1}[0, \infty)$ and $\zeta_{1}, \zeta_{2}, \zeta>0$. Then,

$$
\begin{aligned}
& I^{\zeta_{1}} I^{\zeta_{2}} h(\tau)=I^{\zeta_{1}+\zeta_{2}} h(\tau), \\
& { }^{R} D^{\zeta} I^{\zeta} h(\tau)=h(\tau) .
\end{aligned}
$$

Lemma 2. Let ${ }^{R} D^{\zeta} h(\tau) \in L^{1}[0, \infty), \zeta>0$. Then,

$$
I^{\zeta R} D^{\zeta} h(\tau)=h(\tau)-\sum_{m=1}^{n} b_{m} \tau^{\zeta-m}, \quad \tau \in[0, \infty),
$$

for some $b_{m} \in \mathbb{R}, m=1,2, \ldots, n, n \in \mathbb{N}$.

2.2. Multivalued Function. In this section, we state some necessary facts on multivalued maps [2, 19-21].

Let $(E,\|\|$.$) and (H,\|\|$.$) be Banach spaces and$ $A: E \longrightarrow P_{c l}(E)$ be a multivalued map. The mapping $A$ is

(i) Convex (closed) if $A(e)$ is convex (closed), for every $e \in E$.

(ii) Completely continuous if $A(B)$ is relatively compact, for every $B \in P_{b}(E)$.

(iii) Upper semicontinuous if $A^{-1}(W)$ is closed subset of $E$, for each closed subset $W \subset E$. That is, the set $\{e \in E: A(e) \subseteq O\}$ is open for all open sets $O \subset E$.

(iv) Lower semicontinuous if $A^{-1}(Z)$ is open subset of $E$, for each open subset $Z \subset E$. In the other words, the set $\{e \in E: A(e) \cap O \neq \varnothing\}$ is open for all open sets $O \subset E$.

(v) Measurable multivalued if for every $e \in E$, the function $\tau \longrightarrow d(e, A(\tau))=\inf \{d(e, a): a \in A(\tau)\}$ is $\mathscr{L}$ - measurable function. 
Definition 3. A multivalued map $A:[0, \infty) \times \mathbb{R} \longrightarrow P(\mathbb{R})$ is known as a Caratheodory if

(1) For all $r \in \mathbb{R}, \tau \longrightarrow A(\tau, r)$ is measurable.

(2) For a.e $\tau \in[0, \infty), r \longrightarrow A(\tau, r)$ is upper semicontinuous.

In addition to assumptions (1) and (2), the map $A$ is $L^{1}$-Caratheodory if for each $k>0, \exists \phi_{k} \in C[0, \infty) \cap L^{1}[0, \infty)$ satisfying $\sup _{\tau \geq 0}\left|\phi_{k}(\tau)\right|<+\infty$ and $\phi_{k}>0$, that is,

$$
\begin{aligned}
\left\|A\left(\tau,\left(1+\tau^{\alpha-1}\right) r\right)\right\|= & \sup \left\{|a|: a \in A\left(\tau,\left(1+\tau^{\alpha-1}\right) r\right)\right\} \\
& \leq \phi_{k}(\tau),
\end{aligned}
$$

for all $|r| \leq k, \tau \in[0, \infty)$.

2.3. Fredholm Operator. We recall some preliminaries about the operators specially Fredholm type where we can get them from [3, 22-24].

Let $L: E \longrightarrow H$ be any considered operator. Then, we define the following.

(1) The kernel of $L$ by

$$
\operatorname{Ker}(L)=\left\{e \in E: L(E)=0_{H}\right\} .
$$

(2) The image (rang) of $L$ by

$$
\operatorname{Im}(L)=R(L)=\{L(e): e \in E\} \subseteq H .
$$

(3) The cokernel of $L$ by

$$
\operatorname{Coker}(L)=\{h: h \in H / \operatorname{Im}(L)\} \text {. }
$$

(4) The index of $F$ by

$$
\operatorname{Ind}(L)=\operatorname{dim} \operatorname{Ker}(L)-\operatorname{dim} \operatorname{Coker}(L) .
$$

Note that

$$
\operatorname{dim} \operatorname{Coker}(L)=\operatorname{codim} \operatorname{Im}(L) .
$$

The operator $L$ is called Fredholm operator if it is a bounded linear operator with finite dimensional kernel and cokernel and closed rang. It is said to be Fredholm of index zero if and only if $\operatorname{Ind}(L)=0$.

Let $L: \operatorname{dom}(L) \subset E \longrightarrow H$ be Fredholm of index zero and $P: E \longrightarrow E, Q: H \longrightarrow H$ be continuous projections. In the case of

$$
\begin{aligned}
\operatorname{Im}(P) & =\operatorname{Ker}(L) \wedge \operatorname{Im}(L)=\operatorname{Ker}(Q), \\
E & =\operatorname{Ker}(L) \oplus \operatorname{Ker}(P) \wedge H=\operatorname{Im}(L) \oplus \operatorname{Im}(Q),
\end{aligned}
$$

then $\left.L\right|_{\text {dom }(L) \cap \operatorname{Ker}(P)}$ is invertible, and so we can define

$$
K_{P}=\left(\left.L\right|_{\operatorname{dom}(L) \cap \operatorname{Ker}(P)}\right)^{-1} \text {. }
$$

In fact, there is an isomorphic map $J: \operatorname{Im}(Q) \longrightarrow \operatorname{Ker}(L)$. Consider the inclusion

$$
L v \in N v,
$$

which is equivalent to

$$
\nu \in(P+J Q N) v+K_{p}(I-Q) N v .
$$

Let $C \subset E$ be a convex and a closed subset. Then, $C$ is called a cone if
(i) $\rho v \in C, \forall v \in C, \rho \geq 0$
(ii) $v,-v \in C \Longrightarrow v=0$

Consequently, there is an induced partial order $\leqslant$ in $E$ subject to

$$
o \preccurlyeq v \Longleftrightarrow v-o \in C \text {. }
$$

Lemma 3. Let $E$ be a Banach space contains a cone $C$. Then, for every $\eta \in C /\{0\}$, there exists a positive number $\sigma(\eta)$ which satisfies

$$
\|\nu+\eta\| \geq \sigma(\eta)\|\nu\|, \quad \nu \in C .
$$

Define $\mu: E \longrightarrow C$ to be a retraction (continuous mapping constrained with $\mu(\eta)=\eta, \forall \eta \in C)$. Define the map $\Theta$ as

$$
\Theta=P+J Q N+K_{p}(I-Q) N \text {. }
$$

Then,
(i) $L v \in N v \Longleftrightarrow v \in(P+J Q N) v+K_{p}(I-Q) N v \Longleftrightarrow$ $\nu \in \Theta \nu$

(ii) $\Theta_{\mu}=\Theta^{\circ} \mu$

Theorem 1. (O'Regan and Zima). Let $C \subset E$ be a cone, and $\Omega_{1}$ and $\Omega_{2}$ be open bounded subsets of $E$ which satisfy $\overline{\Omega_{1}} \subset \Omega_{2}$, and $C \cap\left(\overline{\Omega_{2}} / \Omega_{1}\right) \neq \varnothing$. Assume that

(1) $L: E \longrightarrow H$ is a Fredholm operator of index zero.

(2) $N: E \longrightarrow 2^{H}$ is an upper-semicontinuous mapping with nonempty compact convex values.

(3) QN: $E \longrightarrow 2^{H}$ is bounded on bounded subsets of $C$, and $K_{p}(I-Q) N: E \longrightarrow 2^{E}$ is compact on every bounded subset of $C$.

(4) $L \nu \notin \gamma N \nu, \forall \nu \in C \cap \partial \Omega_{2} \cap \operatorname{domL}$ and $\gamma \in(0,1)$.

(5) $\mu$ subsets of $\overline{\Omega_{2}}$ into bounded subsets of $C$.

(6) $\operatorname{deg}\left(\left[I-(P+J Q N)_{\mu}\right]_{\text {KerL }}, \operatorname{KerL} \cap \Omega_{2}, 0\right) \neq 0$.

(7) There is $v_{0} \in C /\{0\}$ such that

$$
\|\nu\| \leq \sigma\left(\nu_{0}\right)\|\eta\|, \quad \forall v \in C\left(\nu_{0}\right) \cap \partial \Omega_{1}, \eta \in \Theta \nu,
$$

where

$$
C\left(\nu_{0}\right)=\left\{\nu \in C \mid \rho v_{0} \preccurlyeq \nu, \quad \text { for some } \rho>0\right\},
$$

and $\sigma\left(\nu_{0}\right)$ is such that $\left\|\nu+v_{0}\right\| \geq \sigma\left(\nu_{0}\right)\|\nu\|, \quad \nu \in C$.

(8) $(P+J Q N)_{\mu}\left(\partial \Omega_{2}\right) \subset C$.

(9) $\Theta_{\mu}\left(\overline{\Omega_{2}} / \Omega_{1}\right) \subset C$.

(10) $\nu \notin(P+J Q N)_{\mu} \nu, \quad \nu \in \operatorname{Ker} L \cap \partial \Omega_{2}$. 
Then, the inclusion $L v \in N v$ can be solvable in the set $C \cap\left(\overline{\Omega_{2}} / \Omega_{1}\right)$.

Remark 1. Let

$$
E=\left\{\nu \mid \nu \in C[0, \infty), \lim _{\tau \longrightarrow \infty} \frac{\nu(\tau)}{1+\tau^{\alpha-1}} \text { exists }\right\},
$$

with the norm

$$
\|\nu\|_{E}=\sup _{\tau \geq 0} \frac{\nu(\tau)}{1+\tau^{\alpha-1}} .
$$

And let

$$
\begin{aligned}
H= & \left\{h \mid h(\tau) \in C[0, \infty) \cap L^{1}[0, \infty) \cap A(\tau, \nu(\tau)),\right. \\
& \left.\sup _{\tau \geq 0}|h(\tau)|<+\infty\right\},
\end{aligned}
$$

with the norm

$$
\|h\|_{H}=\sup _{\tau \geq 0}|h(\tau)|+\int_{0}^{\infty}|h(\tau)| \mathrm{d} \tau .
$$
[11].

Then, $\left(E,\|\cdot\|_{E}\right)$ and $\left(H,\|\cdot\|_{H}\right)$ are Banach spaces, see

Expand the operator $L$, for which

$$
\begin{aligned}
\operatorname{dom} L= & \left\{\nu \in E \mid{ }^{R} D^{\alpha}[\kappa(\tau) \nu(\tau)] \in C[0, \infty) \cap L^{1}[0, \infty),\right. \\
& v^{(i)}(0)=0, i=0, . ., n-2, \\
& \left.{ }^{R} D^{\alpha-1}[\kappa(\tau) \nu(\tau)]_{\tau=0}=\lim _{\tau \longrightarrow \infty}{ }^{R} D^{\alpha-1}[\kappa(\tau) \nu(\tau)]\right\},
\end{aligned}
$$

where

$$
L: \operatorname{domL} \subset E \longrightarrow H, \quad \nu \mapsto^{R} D^{\alpha}[\kappa(\tau) \nu(\tau)],
$$

$N: E \longrightarrow H, \quad \nu \longmapsto a(\tau, \nu(\tau))$

Then, the multivalue problems (1)-(3) are equivalent to the inclusion:

$$
L v \in N v, \quad v \in \text { domL. }
$$

We can understand the proof of the following lemma if we see the compactness hypothesis in $[11,25,26]$.

Lemma 4. Let $\Omega$ be subset of $E$; then, $\Omega$ is a relatively compact subset if and only if the next conditions hold.

(i) The subset $\Omega$ is uniformly bounded, i.e.,

$$
\forall \nu \in \Omega, \quad \exists R>0 \ni\|\nu\|_{E} \leq R .
$$

(ii) Let I be compact subinterval of $[0, \infty)$ and let for all $\epsilon>0$ there exists $\delta=\delta(\epsilon)>0$ such that $\left|\tau_{1}-\tau_{2}\right|<\delta$ for $\tau_{1}, \tau_{2} \in I$. Then,

$$
\left|\frac{\nu\left(\tau_{1}\right)}{1+\tau_{1}^{\alpha-1}}-\frac{\nu\left(\tau_{2}\right)}{1+\tau_{2}^{\alpha-1}}\right|<\epsilon, \quad \forall v \in \Omega,
$$

(iii) For $\epsilon>0$ be given, there exists $\lambda=\lambda(\epsilon)$ in since

$$
\left|\frac{v\left(\omega_{1}\right)}{1+\omega_{1}^{\alpha-1}}-\frac{v\left(\omega_{2}\right)}{1+\omega_{2}^{\alpha-1}}\right|<\epsilon, \quad \forall v \in \Omega, \omega_{1}, \omega_{2}>\lambda .
$$

\section{Acquired Upshots}

In this section, we discuss some results for the sake of seeing the attainability of solution by apply (O'Regan and Zima, Theorem 1) on the half line. Consequently, in view of the arguments of Fredholm operator, we first need to explore some lemmas and facts as follows.

3.1. Basic Requirements. Here, by using the general form of solution, we prove several facts in order to explore that

$\left(\mathscr{H}_{1}\right) L$ is Fredholm operator of index zero.

Also, we explain that this operator is invertible in the set do $m L \cap \operatorname{KerP}$ to compute the formula of its inverse $K_{p}$. Finally, under the version of Lemma 3 (i) and by the known maps $J, K_{p}, Q$, and $I$, we calculate the operator $J Q+K_{P}(I-$ $Q)$ via green functions and show some related inequalities.

Lemma 5. Consider $h(\tau) \in H$. Then, $v \in E$ is a solution of the boundary value problem:

$$
\begin{aligned}
{ }^{R} D^{\alpha}[\kappa(\tau) \nu(\tau)] & =h(\tau), \quad \tau \in[0, \infty), \\
\nu^{(i)}(0) & =0, \quad i=0, \ldots, n-2, \\
{ }^{R} D^{\alpha-1}[\kappa(0) \nu(0)] & =\lim _{\tau \longrightarrow \infty}{ }^{R} D^{\alpha-1}[\kappa(\tau) \nu(\tau)],
\end{aligned}
$$

if and only if

$$
\nu(\tau)=b \frac{\tau^{\alpha-1}}{\kappa(\tau)}+\frac{1}{\Gamma(\alpha) \kappa(\tau)} \int_{0}^{\tau}(\tau-t)^{\alpha-1} h(t) \mathrm{d} t,
$$

where $b \in \mathbb{R}$ and

$$
\int_{0}^{\infty} h(\tau) \mathrm{d} \tau=0
$$

Proof. The proof can be completely seen if we are backing to both Lemmas 1 and 2 and applying accompanying conditions.

Now, using (33)-(35), we obtain

$$
\begin{aligned}
L \nu & ={ }^{R} D^{\alpha}[\kappa(\tau) \nu(\tau)], \\
N \nu & =\{a \mid a(\tau, \nu(\tau))=q(\tau) \beta(\tau, \nu(\tau)), \beta(\tau) \in B(\tau, \nu(\tau))\},
\end{aligned}
$$

which implies that

$$
\begin{aligned}
& \operatorname{Ker} L=\left\{\nu \mid \nu=b\left(\frac{\tau^{\alpha-1}}{\kappa(\tau)}\right), \quad \tau \geq 0, b \in \mathbb{R}\right\}, \\
& \operatorname{ImL}=\left\{h(\tau) \in H \mid \int_{0}^{\infty} h(\tau) \mathrm{d} \tau=0\right\} \subset H .
\end{aligned}
$$

By using the equation,

$$
\kappa(\tau) \nu(\tau)=b \tau^{\alpha-1}
$$


we can define a continuous projection $P$ on $E$ as in the next Lemma.

Lemma 6. Let $P: E \longrightarrow E$ be a map defined as

$$
P \nu(\tau)=\left[\frac{1}{\Gamma(\alpha)} \int_{0}^{\infty} e^{-t} \kappa(t) \nu(t) \mathrm{d} t\right]\left(\frac{\tau^{\alpha-1}}{\kappa(\tau)}\right) .
$$

Then, $P$ is a continuous projection on $E$.

Proof. Let $v \in E$ and $P$ be defined as in (46). Then,

$$
\begin{aligned}
P^{2} \nu(\tau) & =P[P \nu(\tau)], \\
& =P\left(\left[\frac{1}{\Gamma(\alpha)} \int_{0}^{\infty} e^{-t} \kappa(t) \nu(t) \mathrm{d} t\right]\left(\frac{\tau^{\alpha-1}}{\kappa(\tau)}\right)\right), \\
& =\left[\frac{1}{\Gamma(\alpha)} \int_{0}^{\infty} e^{-t} \kappa(t) \nu(t) \mathrm{d} t\right] P\left(\frac{\tau^{\alpha-1}}{\kappa(\tau)}\right), \\
& =\left[\frac{1}{\Gamma(\alpha)} \int_{0}^{\infty} e^{-t} \kappa(t) \nu(t) \mathrm{d} t\right]\left(\frac{\tau^{\alpha-1}}{\kappa(\tau)}\right), \\
& =P \nu(\tau),
\end{aligned}
$$

which completes the proof.

Remark 2. We can recall that

$$
\begin{aligned}
\operatorname{Ker} P & =\left\{\nu \mid \int_{0}^{\infty} e^{-t} \kappa(t) \nu(t) \mathrm{d} t=0\right\}, \\
\operatorname{Im} P & =\operatorname{Ker} L .
\end{aligned}
$$

Immediately, we have

$$
E=\operatorname{Ker} P \oplus \operatorname{Im} P=\operatorname{Ker} P \oplus \operatorname{Ker} L .
$$

According to both integrals $\int_{0}^{\infty} e^{-t} \mathrm{~d} t=1$ and $\int_{0}^{\infty} h(t) \mathrm{d} t=0$ in (41), we can explain and prove that there exists a continuous projection $Q$ on the space $H$. See the next lemma.

Lemma 7. Let $Q: H \longrightarrow H$ be a mapping formed by

$$
Q h(\tau)=e^{-\tau} \int_{0}^{\infty} h(t) \mathrm{d} t,
$$

where $\tau \geq 0, h(\tau) \in H$. Then, $Q$ is a continuous projection on $H$.

Remark 3. We can make sure that

$$
\begin{aligned}
\operatorname{Im} Q & =\left\{b e^{-\tau}, \quad b \in \mathbb{R}, \tau \geq 0\right\}, \\
\operatorname{Ker} Q & =\operatorname{Im} L .
\end{aligned}
$$

Claim that $H=\operatorname{Ker} Q \oplus \operatorname{Im} Q=\operatorname{Im} L \oplus \operatorname{Im} Q$. For that, take $h(\tau) \in H$. Then, we have

$$
h(\tau)=h(\tau)-Q h(\tau)+Q h(\tau)=(I-Q) h(\tau)+Q h(\tau),
$$

where $\mathrm{Qh}(\tau) \in \operatorname{ImQ}$. Moreover,

$$
\begin{aligned}
Q[(I-Q) h(\tau)] & =Q h(\tau)-Q^{2} h(\tau), \\
& =Q h(\tau)-Q h(\tau)
\end{aligned}
$$

Thus, $\quad H=\operatorname{Ker} Q+\operatorname{Im} Q$. Assume that $h(\tau) \epsilon$ $\operatorname{KerQ} \cap \operatorname{ImQ}$. Then,

$$
\begin{aligned}
h(\tau) & =b e^{-\tau}, \\
Q h(\tau) & =0, \\
\Longrightarrow b e^{-\tau} \int_{0}^{\infty} e^{-\tau} \mathrm{d} \tau & =0, \\
\Longrightarrow b & =0, \\
\Longrightarrow h(\tau) & =0 .
\end{aligned}
$$

Hence, $H=\operatorname{Im} L \oplus \operatorname{ImQ}$. It remains to prove that $L$ is of index zero. Due to the previous results of $Q$, we find that

$1=\operatorname{dim} \mathbb{R}=\operatorname{dim} \operatorname{Im} Q=\operatorname{dim} \operatorname{Coker} \mathrm{Q}=\operatorname{codim} \operatorname{Im} L<+\infty$.

Since $\operatorname{Im} L$ is closed in $H$, it implies that $L$ is the Fredholm operator of index zero.

Furthermore, we can see clearly that $\operatorname{Ker} L \cong \operatorname{ImQ}$ if we define the isomorphism $J: \operatorname{ImQ} \longrightarrow \operatorname{Ker} L$ by

$$
J\left(b e^{-\tau}\right)=b\left(\frac{\tau^{\alpha-1}}{\kappa(\tau)}\right), \quad \tau \geq 0, b \in \mathbb{R},
$$

as long as $\operatorname{ImQ}$ defined by (52).

Once we have $L$ is the Fredholm operator of index zero, then it is invertible in the set $\operatorname{dom} L \cap \operatorname{KerP}$. Create $\left(\left.L\right|_{\text {domLnKerP }}\right)=L_{P}: \operatorname{do} m L \cap \operatorname{Ker} P \longrightarrow \operatorname{ImL}$ and $L_{P}^{-1}=K_{P}$. Let $\nu(\tau) \in$ do $m L \cap \operatorname{Ker} P$, which follows that $\nu(\tau)$ is defined by (40) and satisfying the condition in (48). By substituting (40) in the equation given in (48), the constant $b$ will be subject to

$$
b=-\frac{1}{\Gamma(\alpha)} \int_{0}^{\infty} e^{-t} \int_{0}^{t} \frac{(t-s)^{\alpha-1}}{\Gamma(\alpha)} h(s) \mathrm{d} s \mathrm{~d} t .
$$

Therefore,

$$
\begin{aligned}
\left(K_{P} h\right)(\tau)= & -\left(\frac{1}{\Gamma(\alpha)} \int_{0}^{\infty} e^{-t} \int_{0}^{t} \frac{(t-s)^{\alpha-1}}{\Gamma(\alpha)} h(s) \mathrm{d} s \mathrm{~d} t\right)\left(\frac{\tau^{\alpha-1}}{\kappa(\tau)}\right) \\
& +\frac{1}{\kappa(\tau)} \int_{0}^{\tau} \frac{(\tau-t)^{\alpha-1}}{\Gamma(\alpha)} h(t) \mathrm{d} t, \quad h(\tau) \in \operatorname{ImL} .
\end{aligned}
$$

Lemma 8. Let $L$ be the Fredholm operator of index zero. That is,

$$
\begin{array}{r}
L \nu={ }^{R} D^{\alpha}[\kappa(\tau) \nu(\tau)], \\
\left(\left.L\right|_{\text {domLnKerP }}\right)=L_{P},
\end{array}
$$

where do $m L, P$ and KerP are defined by (32), (46), and (48), respectively. Let $K_{P}$ be elected such as in (60). Then,

$$
K_{P}\left(L_{P}\right)=L_{P}\left(K_{P}\right)=I \text {. }
$$


Proof. Opting $\nu(\tau) \in$ do $m L \cap K \operatorname{Ker} P$ drives

$$
\begin{aligned}
\left(K_{P} L_{P}\right)(\nu)(\tau)= & K_{P}\left(L_{P} \nu\right)(\tau) \\
= & -\left(\frac{1}{\Gamma(\alpha)} \int_{0}^{\infty} e^{-t} \int_{0}^{t} \frac{(t-s)^{\alpha-1}}{\Gamma(\alpha)} L \nu(s) \mathrm{d} s \mathrm{~d} t\right)\left(\frac{\tau^{\alpha-1}}{\kappa(\tau)}\right) \\
& +\frac{1}{\kappa(\tau)} \int_{0}^{\tau} \frac{(\tau-t)^{\alpha-1}}{\Gamma(\alpha)} L \nu(t) \mathrm{d} t \\
= & -\left(\frac{1}{\Gamma(\alpha)} \int_{0}^{\infty} e_{0}^{-t} \frac{(t-s)^{\alpha-1}}{\Gamma(\alpha)} D^{\alpha}[\kappa(s) \nu(s)] \mathrm{d} s \mathrm{~d} t\right) \\
& \times\left(\frac{\tau^{\alpha-1}}{\kappa(\tau)}\right)+\left(\frac{b}{\Gamma(\alpha)} \int_{0}^{\infty} e^{-t} t^{\alpha-1} \mathrm{~d} t\right)\left(\frac{\tau^{\alpha-1}}{\kappa(\tau)}\right) \\
& +v(\tau)-b\left(\frac{\tau^{\alpha-1}}{\kappa(\tau)}\right) \\
= & \nu(\tau) .
\end{aligned}
$$

And use (43) to verify that
$\left(L_{P} K_{P}\right)(h)(\tau)=L_{P}\left(K_{P} h\right)(\tau)$

$$
\begin{aligned}
= & L\left[-\left(\frac{1}{\Gamma(\alpha)} \int_{0}^{\infty} e^{-t} \int_{0}^{t} \frac{(t-s)^{\alpha-1}}{\Gamma(\alpha)} h(s) \mathrm{d} s \mathrm{~d} t\right)\left(\frac{\tau^{\alpha-1}}{\kappa(\tau)}\right)\right. \\
& \left.+\frac{1}{\kappa(\tau)} \int_{0}^{\tau} \frac{(\tau-t)^{\alpha-1}}{\Gamma(\alpha)} h(t) \mathrm{d} t\right] \\
= & { }^{R} D^{\alpha}\left[\kappa(\tau) \frac{1}{\kappa(\tau)} \int_{0}^{\tau} \frac{(\tau-t)^{\alpha-1}}{\Gamma(\alpha)} h(t) \mathrm{d} t\right] \\
= & h(\tau) .
\end{aligned}
$$

Therefore, $K_{P}\left(L_{P}\right)=L_{P}\left(K_{P}\right)=I$.

According to Lemma 3 (i), we need to compute the value:

$$
\left[J Q+K_{P}(I-Q)\right] h(\tau), \quad h(\tau) \in N(\nu),
$$

which can be introduced by

$$
\left[J Q+K_{P}(I-Q)\right] h(\tau)=\left\{h^{*} \in H \mid h^{*}(\tau)=\frac{\tau^{\alpha-1}}{\Gamma(\alpha) \kappa(\tau)}\left[\int_{0}^{\infty} G_{1}(\tau, t) h(t) \mathrm{d} t-\int_{0}^{\infty} \int_{0}^{\tau} G_{2}(\tau, t) h(t) \mathrm{d} t \mathrm{~d} \tau\right], h(t) \in N(\nu)\right\},
$$

where

$$
\begin{aligned}
& G_{1}(\tau, t)= \begin{cases}0, & \tau=0, \\
\Gamma(\alpha)-\int_{0}^{\tau} \frac{(\tau-z)^{\alpha-1}}{\tau^{\alpha-1}} e^{-z} \mathrm{~d} z+\int_{0}^{\infty} \int_{0}^{t} G_{2}(t, m) e^{-m} \mathrm{~d} m \mathrm{~d} t+\frac{(\tau-t)^{\alpha-1}}{\tau^{\alpha-1}}, & \tau \neq 0,0 \leq t \leq \tau<+\infty, \\
\Gamma(\alpha)-\int_{0}^{\tau} \frac{(\tau-z)^{\alpha-1}}{\tau^{\alpha-1}} e^{-z} \mathrm{~d} z+\int_{0}^{\infty} \int_{0}^{t} G_{2}(t, m) e^{-m} \mathrm{~d} m \mathrm{~d} t, & 0<\tau \leq t<+\infty,\end{cases} \\
& G_{2}(\tau, t)= \begin{cases}0, & 0<\tau \leq t<+\infty \\
\frac{(\tau-t)^{\alpha-1}}{\tau^{\alpha-1}} e^{-\tau}, & 0 \leq t \leq \tau<+\infty\end{cases}
\end{aligned}
$$

Lemma 9. We have

$$
\begin{aligned}
0 & \leq G_{2}(\tau, t) \leq \frac{\tau^{\alpha-1}}{\Gamma(\alpha)} e^{-\tau} \\
0 & <\Gamma(\alpha)-1+\frac{1}{2^{\alpha+1}} \leq G_{1}(\tau, t) \leq 1+\alpha+\Gamma(\alpha), \quad \forall \alpha>1 .
\end{aligned}
$$

Proof. Basically, we have the following inequalities:

$$
\begin{aligned}
& 0<\int_{0}^{\tau} \frac{(\tau-z)^{\alpha-1}}{\tau^{\alpha-1}} e^{-z} d z \leq \int_{0}^{\infty} e^{-z} \mathrm{~d} z=1, \\
& 0 \leq G_{2}(\tau, t) \leq \frac{\tau^{\alpha-1}}{\Gamma(\alpha)} e^{-\tau} .
\end{aligned}
$$

In order to prove the L.H.S of (69), we note that, for all positive reals $m<t \Longrightarrow e^{-m}>e^{-t}$, to imply that

$$
\begin{aligned}
\int_{0}^{\infty} \int_{0}^{t} G_{2}(t, m) e^{-m} \mathrm{~d} m \mathrm{~d} t & \geq \int_{0}^{\infty} \int_{0}^{t} G_{2}(t, m) e^{-t} \mathrm{~d} m \mathrm{~d} t \\
& =\frac{1}{\Gamma(\alpha+1)} \int_{0}^{\infty} t^{\alpha} e^{-2 t} \mathrm{~d} t=\frac{1}{2^{\alpha+1}},
\end{aligned}
$$

which leads to

$$
\Gamma(\alpha)-1+\frac{1}{2^{\alpha+1}} \leq G_{1}(\tau, t) .
$$

By analytical methods, we can see that 


$$
0<\Gamma(\alpha)-1+\frac{1}{2^{\alpha+1}}, \quad \forall \alpha>1
$$

Now, to make sure about the R.H.S of (69), use

$$
-\int_{0}^{\tau} \frac{(\tau-z)^{\alpha-1}}{\tau^{\alpha-1}} e^{-z} \mathrm{~d} z<0
$$

Then,

$$
\begin{aligned}
G_{1}(\tau, t) & \leq \Gamma(\alpha)+1+\int_{0}^{\infty} \int_{0}^{t} G_{2}(t, m) e^{-m} \mathrm{~d} m \mathrm{~d} t \\
& \leq \Gamma(\alpha)+1+\int_{0}^{\infty} \frac{t^{\alpha-1}}{\Gamma(\alpha)} e^{-t} \int_{0}^{t} e^{-m} \mathrm{~d} m \mathrm{~d} t \\
& \leq \Gamma(\alpha)+1+\int_{0}^{\infty} \frac{t^{\alpha}}{\Gamma(\alpha)} e^{-t} \mathrm{~d} t \\
& =\Gamma(\alpha)+1+\alpha .
\end{aligned}
$$

3.2. Essential Theorem. Apply all results in the first part to illustrate the main theorem with some needed hypothesis as follows.

Theorem 2. Reckoning in addition of $\left(\mathscr{H}_{1}\right)$ that the following.

$\left(\mathscr{H}_{2}\right) \quad$ Let $B:[0 . \infty) \times \mathbb{R} \longrightarrow B C C(\mathbb{R})$ be $L^{1}-$ Caratheodory (Definition 3) with $S_{B, v}$ which has nonempty compact convex values conditional on which there exist nonnegative functions $\gamma_{i}(\tau), i=1,2,3, \eta_{j}(\tau), j=1,2$ and $\theta(\tau)$ satisfying

$$
\begin{gathered}
\beta(\tau, \nu(\tau)) \leq-\gamma_{1}(\tau)|\beta(\tau, \nu(\tau))|+\gamma_{2}(\tau) \frac{\nu(\tau)}{1+\tau^{\alpha-1}}+\gamma_{3}(\tau) \\
-\theta(\tau) \frac{\nu(\tau)}{1+\tau^{\alpha-1}} \leq \beta(\tau, \nu(\tau)) \leq-\eta_{1}(\tau) \frac{\nu(\tau)}{1+\tau^{\alpha-1}}+\eta_{2}(\tau)
\end{gathered}
$$

for all $\beta(\tau) \in B(\tau, \nu(\tau))$, and $0<\nu(\tau) / 1+\tau^{\alpha-1}<R$.

$$
\begin{gathered}
\left(\mathscr{H}_{3}\right) \text { For } a(\tau, \nu(\tau))=q(\tau) \beta(\tau, \nu(\tau)), \text { we have } \\
\int_{0}^{\infty} q(\tau) \theta(\tau) \mathrm{d} \tau<\frac{\Gamma(\alpha)+1}{2(\Gamma(\alpha)+1 / 2)(\Gamma(\alpha)+\alpha+1)}, \\
e^{\tau} q(\tau) \theta(\tau)<\frac{1+\tau^{\alpha-1}}{(\Gamma(\alpha)+\alpha+1)}
\end{gathered}
$$

and there exists $\delta>0$ in which

$$
\delta=\frac{\Gamma(\alpha)}{\Gamma(\alpha)+(1 / 2)}+\frac{(\Gamma(\alpha)+\alpha+1))}{\Gamma(\alpha)+1} \int_{0}^{\infty} q(\tau) \theta(\tau) \mathrm{d} \tau<1 .
$$

$\left(\mathscr{H}_{4}\right)$ There exist positive constants $q_{0}, \gamma_{0}, \Gamma_{0}$, and $R_{0}$, where as

$$
\Gamma_{0}=\frac{2}{\gamma_{0}} \sup _{\tau \geq 0}\left[\frac{\gamma_{2}(\tau)+\left(e^{-\tau} \kappa(\tau) \gamma_{0}\left(1+\tau^{\alpha-1}\right)\right) / 2 q(\tau)}{\eta_{1}(\tau)}\right],
$$

$$
\begin{aligned}
R_{0}= & \max \left\{\frac{q_{0} \Gamma_{0}}{\Gamma(\alpha)} \int_{0}^{\infty} \eta_{2}(\tau) \mathrm{d} \tau, \frac{2 q_{0}}{\gamma_{0} \Gamma(\alpha)} \int_{0}^{\infty} \gamma_{3}(\tau) \mathrm{d} \tau,\right. \\
& \left.\frac{q_{0}}{\eta_{0}} \int_{0}^{\infty} \eta_{2}(\tau) \mathrm{d} \tau\right\},
\end{aligned}
$$

where $q_{0}=\sup _{\tau \geq 0}|q(\tau)|, \gamma_{0}=\inf _{\tau \geq 0} \gamma_{1}(\tau)$, and

$$
\eta_{0}=\int_{0}^{\infty} q(t) \eta_{1}(t) \frac{t^{\alpha-1}}{\left(1+t^{\alpha-1}\right) \kappa(t)} \mathrm{d} t \text {. }
$$

Then, it is distinctly possible to find positive solution for problems (1)-(3) in $C \cap\left(\overline{\Omega_{2}} / \Omega_{1}\right)$.

Proof. Let $E$ and $H$ be Banach spaces defined as in Remark. Define the linear operator $L$ and its property by (32), (33), (43), and (44), and the nonlinear operator $N$ by (34) as well as (35) holds. Then, to show that if it is possible for the given problem to has a solution, we will prove all points of (O’Regan and Zima, Theorem 1-).

Step 1: the operator $L$ will be the Fredholm operator of index zero by using $\left(\mathscr{H}_{1}\right)$.

Step 2: by $\left(\mathscr{H}_{2}\right)$, the operator $(N)$ is upper semicontinuous mapping with nonempty compact convex values.

Step 3: according to $\left(\mathscr{H}_{2}\right), Q N$ is bounded in the bounded set and

$$
K_{P}(I-Q) N
$$

is compact, where $P, Q$, and $K_{P}$ are all conformed by (46), (51), and (60), respectively.

Define the needed sets $\Omega_{1}, \Omega_{2}$, and $C$ as follows:

$$
\begin{aligned}
\Omega_{1} & =\left\{\nu \in E \mid \epsilon\|\nu\|_{E}<\frac{\nu(\tau)}{1+\tau^{\alpha-1}}<R_{1}, R_{1} \in\left(0, R_{2}\right), \epsilon \in(\delta, 1)\right\}, \\
\Omega_{2} & =\left\{\nu \in E \mid\|v\|_{E}<R_{2}, R_{2} \in\left(R_{0}, R\right)\right\}, \\
C & =\{\nu \in E \mid \nu(\tau) \geq 0, \tau \geq 0\} \subset E .
\end{aligned}
$$

Clearly, $\overline{\Omega_{1}} \subset \Omega_{2}$ and $C$ forms a cone in $E$.

Step 4: claim that $L \nu \notin \gamma N \nu, \forall \nu \in C \cap \partial \Omega_{2} \cap$ do $m L, \gamma \in(0,1)$.

Assume by contradiction that $v \geq 0,\|v\|_{E}=R_{2}$ such that $L v \in \gamma N \nu$. Since

$$
\begin{aligned}
\nu & =v-P \nu+P v=K_{P} L(I-P) \nu+P v=K_{P} L v+P v, \\
L v & =\gamma h(\tau), \\
h(\tau) & \in N v,
\end{aligned}
$$


we have, by using Hölder's inequality with $p=1$ and $q=\infty$,

(66) and (68) that

$$
\begin{aligned}
\frac{\nu(\tau)}{1+\tau^{\alpha-1}}= & \frac{\tau^{\alpha-1}}{\Gamma(\alpha) \kappa(\tau)\left(1+\tau^{\alpha-1}\right)}\left[\int_{0}^{\infty} e^{-t} \kappa(t) \nu(t) \mathrm{d} t-\int_{0}^{\infty} e^{-t} I^{\alpha R} D^{\alpha}[\kappa(t) \nu(t)] \mathrm{d} t\right] \\
& +\frac{1}{\kappa(\tau)\left(1+\tau^{\alpha-1}\right)} I^{\alpha R} D^{\alpha}[\kappa(\tau) \nu(\tau)] \\
\leq & \frac{1}{\Gamma(\alpha)} \int_{0}^{\infty} e^{-t} \kappa(t) \nu(t) \mathrm{d} t \\
& +\frac{\tau^{\alpha-1}}{\Gamma(\alpha) \kappa(\tau)\left(1+\tau^{\alpha-1}\right)}\left[\frac{1}{\Gamma(\alpha)} \int_{0}^{\infty} e^{-t} t^{\alpha-1} \int_{0}^{t}{ }^{R} D^{\alpha}[\kappa(s) \nu(s)] \mathrm{d} s \mathrm{~d} t+\int_{0}^{\infty}{ }^{R} D^{\alpha}[\kappa(t) \nu(t)] \mathrm{d} t\right] \\
\leq & \frac{1}{\Gamma(\alpha)} \int_{0}^{\infty} e^{-t} \kappa(t) \nu(t) \mathrm{d} t+\frac{2}{\Gamma(\alpha)} \int_{0}^{\infty}\left|R D^{\alpha}[\kappa(t) \nu(t)]\right| \mathrm{d} t .
\end{aligned}
$$

In case we can see by $\left(\mathscr{H}_{2}(76)\right.$ and (77)) that

${ }^{R} D^{\alpha}[\kappa(\tau) \nu(\tau)]=L \nu=\gamma a(\tau, \nu(\tau))=\gamma q(\tau) \beta(\tau, \nu(\tau))$,

$$
\begin{aligned}
{ }^{R} D^{\alpha}[\kappa(\tau) \nu(\tau)] & =\gamma q(\tau) \beta(\tau, \nu(\tau)) \\
& \leq-\gamma \gamma_{1}(\tau) q(\tau)|\beta(\tau, \nu(\tau))|+\gamma \gamma_{2}(\tau) q(\tau) \frac{\nu(\tau)}{1+\tau^{\alpha-1}}+\gamma \gamma_{3}(\tau) q(\tau) \\
& \leq-\left.\gamma_{1}(\tau)\right|^{R} D^{\alpha}[\kappa(\tau) \nu(\tau)] \mid+\gamma_{2}(\tau) q(\tau) \frac{\nu(\tau)}{1+\tau^{\alpha-1}}+\gamma_{3}(\tau) q(\tau), \\
{ }^{R} D^{\alpha}[\kappa(\tau) \nu(\tau)]= & \gamma q(\tau) \beta(\tau, \nu(\tau)) \\
\leq & -\gamma \eta_{1}(\tau) q(\tau) \frac{\nu(\tau)}{1+\tau^{\alpha-1}}+\gamma q(\tau) \eta_{2}(\tau) .
\end{aligned}
$$

Now, we have

Relations (89), (90), and (91) give the ensuing results:

$\int_{0}^{\infty}{ }^{R} D^{\alpha}[\kappa(t) \nu(t)] \mathrm{d} t=\int_{0}^{\infty} D^{R} D^{\alpha-1}[\kappa(t) \nu(t)] \mathrm{d} t$

$=\lim _{t \longrightarrow \infty}{ }^{R} D^{\alpha-1}[\kappa(t) \nu(t)]-\left.{ }^{R} D^{\alpha-1}[\kappa(t) \nu(t)]\right|_{t=0}=0$.

$$
\begin{aligned}
0= & \int_{0}^{\infty}{ }^{R} D^{\alpha}[\kappa(t) \nu(t)] \mathrm{d} t \leq-\left.\int_{0}^{\infty} \gamma_{1}(t)\right|^{R} D^{\alpha}[\kappa(t) \nu(t)] \mid \mathrm{d} t \\
& +\int_{0}^{\infty} \gamma_{2}(t) q(t) \frac{\nu(t)}{1+t^{\alpha-1}} \mathrm{~d} t+\int_{0}^{\infty} \gamma_{3}(t) q(t) \mathrm{d} t, \\
0= & \int_{0}^{\infty}{ }^{R} D^{\alpha}[\kappa(t) \nu(t)] \mathrm{d} t \\
& \leq-\int_{0}^{\infty} \gamma \eta_{1}(t) q(t) \frac{\nu(t)}{1+t^{\alpha-1}} \mathrm{~d} t+\int_{0}^{\infty} \gamma \eta_{2}(t) q(t) \mathrm{d} t,
\end{aligned}
$$


that are equivalent, respectively, to

$$
\begin{gathered}
\int_{0}^{\infty}\left|{ }^{R} D^{\alpha}[\kappa(t) \nu(t)]\right| \mathrm{d} t \leq \frac{1}{\gamma_{0}} \int_{0}^{\infty} \gamma_{2}(t) q(t) \frac{\nu(t)}{1+t^{\alpha-1}} \mathrm{~d} t+\frac{1}{\gamma_{0}} \int_{0}^{\infty} \gamma_{3}(t) q(t) \mathrm{d} t \\
\int_{0}^{\infty} \eta_{1}(t) q(t) \frac{v(t)}{1+t^{\alpha-1}} \mathrm{~d} t \leq \int_{0}^{\infty} \eta_{2}(t) q(t) \mathrm{d} t .
\end{gathered}
$$

From (95), it can be seen that

$$
\int_{0}^{\infty} q(t) \eta_{1}(t) \frac{v(t)}{1+t^{\alpha-1}} \mathrm{~d} t \leq q_{0} \int_{0}^{\infty} \eta_{2}(t) \mathrm{d} t .
$$

The relations (81) and (94)-(96) together with $\left(\mathscr{H}_{4}\right)$ show that (87) will be equivalent to

$$
\begin{aligned}
\frac{v(\tau)}{1+\tau^{\alpha-1}} \leq & \frac{2 q_{0}}{\gamma_{0} \Gamma(\alpha)} \int_{0}^{\infty} \gamma_{2}(t) \frac{v(t)}{1+t^{\alpha-1}} \mathrm{~d} t \\
& +\frac{2 q_{0}}{\gamma_{0} \Gamma(\alpha)} \int_{0}^{\infty} \gamma_{3}(t) \mathrm{d} t+\int_{0}^{\infty} e^{-t} \kappa(t) \nu(t) \mathrm{d} t \\
\leq & \frac{\Gamma_{0}}{\Gamma(\alpha)} \int_{0}^{\infty} q(t) \eta_{1}(t) \frac{v(t)}{1+t^{\alpha-1}} \mathrm{~d} t+\frac{2 q_{0}}{\gamma_{0} \Gamma(\alpha)} \int_{0}^{\infty} \gamma_{3}(t) \mathrm{d} t \\
\leq & \frac{q_{0} \Gamma_{0}}{\Gamma(\alpha)} \int_{0}^{\infty} \eta_{2}(t) \mathrm{d} t+\frac{2 q_{0}}{\gamma_{0} \Gamma(\alpha)} \int_{0}^{\infty} \gamma_{3}(t) \mathrm{d} t \\
< & R_{0}<R_{2}<R,
\end{aligned}
$$

which is contradiction to $v \in C \cap \partial \Omega_{2} \cap \operatorname{domL}$.

Step 5: to recall that $\mu$ maps subsets of $\overline{\Omega_{2}}$ into bounded sets of $C$, let $\nu \in \overline{\Omega_{2}}$. Since $\mu(\nu(\tau))=|\nu(\tau)|=$ $\nu(\tau), \forall v \in C$, then

$$
\|\mu(\nu)\|_{C}=\|\mu(\nu)\|_{E}=\|\nu\|_{E} \leq R_{2}, \quad \forall v \in \overline{\Omega_{2}} .
$$

Step 6: this step will show that

$$
\operatorname{deg}\left(\left[I-(P+J Q N)_{\mu}\right]_{\text {KerL }}, \operatorname{Ker} L \cap \Omega_{2}, 0\right) \neq 0 .
$$

Define the map $\hat{H}(\nu, \rho)$ by

$$
\widehat{H}(\nu, \rho)=\left[I-\rho(P+J Q N)_{\mu}\right] \nu, \quad \rho \in[0,1] .
$$

Let $v \in \operatorname{Ker} L \cap \overline{\Omega_{2}}$, and consider by contradiction that $0 \in \hat{H}(\nu, \rho)$. Since $\nu \in \operatorname{Ker} L$, then it is given by the form

$$
\nu(\tau)=b \frac{\tau^{\alpha-1}}{\kappa(\tau)}, \quad \tau \geq 0, b \in \mathbb{R} .
$$

Consequently, we have

$$
\begin{aligned}
\widehat{H}(\nu, \rho) & =\left[I-\rho(P+J Q N)_{\mu}\right]\left(b \frac{\tau^{\alpha-1}}{\kappa(\tau)}\right) \\
& =\left[b-\rho|b|-\rho \int_{0}^{\infty} q(t) f\left(t,|b| \frac{t^{\alpha-1}}{\kappa(t)}\right) \mathrm{d} t\right] \frac{\tau^{\alpha-1}}{\kappa(\tau)},
\end{aligned}
$$

where $b \in\left[-R_{2}, R_{2}\right], \rho \in(0,1]$. Now, expound a map

$$
J_{1}: \operatorname{Ker} L \cap \overline{\Omega_{2}} \longrightarrow \mathbb{R},
$$

such as

$$
J_{1}\left(b \frac{\tau^{\alpha-1}}{\kappa(\tau)}\right)=b
$$

Then, $J_{1}, J(J$ as in (58)) are used to see that

$$
\begin{aligned}
d_{B} & {\left[\widehat{H}\left(b \frac{\tau^{\alpha-1}}{\mathcal{\kappa}(\tau)}, \rho\right), \operatorname{Ker} L \cap \Omega_{2}, 0\right] } \\
& =d_{B}\left[J_{1} \hat{H}\left(J_{1}^{-1} b, \rho\right), J_{1}\left(\operatorname{Ker} L \cap \Omega_{2}\right), 0\right] .
\end{aligned}
$$

so that

$$
0 \in J_{1} \widehat{H}\left(J_{1}^{-1} b, \rho\right) .
$$

Thus, for all $a(\tau, \nu(\tau))=q(\tau) \beta(\tau, \nu(\tau)) \in A(\tau, \nu(\tau))$, we have

$$
\begin{aligned}
& b-\rho|b|-\rho \int_{0}^{\infty} q(t) \beta\left(t,|b| \frac{t^{\alpha-1}}{\kappa(t)}\right) \mathrm{d} t=0 \\
& \Longleftrightarrow b=\rho\left(|b|+\int_{0}^{\infty} q(t) \beta\left(t,|b| \frac{t^{\alpha-1}}{\kappa(t)}\right) \mathrm{d} t\right) .
\end{aligned}
$$

Using $\left(\mathscr{H}_{2}(77)\right)$ and $\left(\mathscr{H}_{3}(78)\right)$ to explain that $b \geq 0$, take $b_{0} \in J_{1}\left(\operatorname{Ker} L \cap \Omega_{2}\right)$, then $\left|b_{0}\right|=R_{2}$. Suppose $0 \in J_{1} \hat{H}\left(J_{1}^{-1}\right.$ $b, \rho)$, which leads to $b_{0}=R_{2}$. Together with $\left(\mathscr{H}_{2}(77)\right)$ and $\left(\mathscr{H}_{4}(82)\right)$, we work out that $\exists a(\tau, \nu(\tau))=q(\tau) \beta\left(\tau, R_{2}\right.$ $\left.\left(\tau^{\alpha-1} / \kappa(\tau)\right)\right) \in A\left(\tau, R_{2}\left(\tau^{\alpha-1} / \kappa(\tau)\right)\right)$, satisfying

$$
\begin{aligned}
R_{2}= & \rho\left(\left|R_{2}\right|+\int_{0}^{\infty} q(t) \beta\left(t,\left|R_{2}\right| \frac{t^{\alpha-1}}{\kappa(t)}\right) \mathrm{d} t\right) \\
\leq & \rho R_{2}\left(1-\int_{0}^{\infty} q(t) \eta_{1}(t) \frac{t^{\alpha-1}}{\left(1+t^{\alpha-1}\right) \kappa(t)} \mathrm{d} t\right) \\
& +\int_{0}^{\infty} q(t) \eta_{2}(t) \mathrm{d} t \\
\leq & \rho R_{2} \leq R_{2} .
\end{aligned}
$$

If $\rho=0$, then $R_{2}=0$ is impossible. This is the cause of $0 \notin J_{1} \widehat{H}\left(J_{1}^{-1} b, \rho\right) \quad$ for $\quad$ all $\quad b \in J_{1}\left(\operatorname{Ker} L \cap \Omega_{2}\right), \rho \in[0,1]$. Therefore, 


$$
\begin{aligned}
\operatorname{deg} & \left(\left[I-(P+J Q N)_{\mu}\right]_{\text {KerL }}, \operatorname{Ker} L \cap \Omega_{2}, 0\right) \\
& =\operatorname{deg}\left(\widehat{H}(., 1), \operatorname{Ker} L \cap \Omega_{2}, 0\right) \\
& =d_{B}\left[J_{1} \hat{H}\left(J_{1}^{-1} b, 1\right), J_{1}\left(\operatorname{Ker} L \cap \Omega_{2}\right), 0\right] \\
& =d_{B}\left[I, J_{1}\left(\operatorname{Ker} L \cap \Omega_{2}\right), 0\right]=1 \neq 0 .
\end{aligned}
$$

Step 7: in this step, our aim is proving that there is $\nu_{0} \in C /\{0\}$, where

$\|\nu\| \leq \sigma\left(v_{0}\right)\|\eta\|, \quad \forall v \in C\left(v_{0}\right) \cap \partial \Omega_{1}, \eta \in \Theta \nu$,

where

$$
C\left(v_{0}\right)=\left\{v \in C \mid \rho v_{0} \leqslant v, \quad \text { for some } \rho>0\right\},
$$

and $\sigma\left(\nu_{0}\right)$ that is $\left\|v+v_{0}\right\| \geq \sigma\left(\nu_{0}\right)\|\nu\|, \nu \in C$.
In order to see that take $\nu_{0}(\tau)=1+\tau^{\alpha-1}, \sigma\left(\nu_{0}\right)=1$, and

$$
C\left(v_{0}\right)=\left\{v \in C \mid \inf _{\tau \geq 0}\left[\frac{v(\tau)}{1+\tau^{\alpha-1}}\right]>0\right\} .
$$

Now, let

$$
\tau_{0}>0 \ni \frac{\tau_{0}^{\alpha-1}}{\kappa\left(\tau_{0}\right)\left(1+\tau_{0}^{\alpha-1}\right)}>\frac{\Gamma(\alpha)+(1 / 2)}{\Gamma(\alpha)+1} .
$$

Then, for $v \in C\left(v_{0}\right) \cap \partial \Omega_{1}$, we have

$$
\left\{\begin{array}{l}
\|\nu\|_{E} \leq R_{1}<R_{2}, \\
\frac{v(\tau)}{1+\tau^{\alpha-1}} \geq \epsilon\|\nu\|_{E}, \quad \epsilon \in(\delta, 1) .
\end{array}\right.
$$

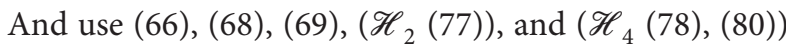
to show that

$$
\begin{aligned}
\frac{(\Theta \nu)\left(\tau_{0}\right)}{1+\tau_{0}^{\alpha-1}} & =\frac{1}{1+\tau_{0}^{\alpha-1}}\left[P \nu\left(\tau_{0}\right)+\left(J Q N+K_{P}(I-Q) N\right) \nu\left(\tau_{0}\right)\right] \\
& =\frac{\tau_{0}^{\alpha-1}}{\Gamma(\alpha) \kappa\left(\tau_{0}\right)\left(1+\tau_{0}^{\alpha-1}\right)}\left[\int_{0}^{\infty} e^{-t} \kappa(t) \nu(t) \mathrm{d} t+\int_{0}^{\infty} G_{1}\left(\tau_{0}, t\right) q(t) \beta(t, \nu(t)) \mathrm{d} t-\int_{0}^{\infty} \int_{0}^{\tau_{0}} G_{2}\left(\tau_{0}, t\right) q(t) \beta(t, \nu(t)) \mathrm{d} t \mathrm{~d} \tau_{0}\right] \\
& \geq \frac{\tau_{0}^{\alpha-1}}{\kappa\left(\tau_{0}\right)\left(1+\tau_{0}^{\alpha-1}\right)}\left[\epsilon\|\nu\|_{E} \frac{1+\Gamma(\alpha)}{\Gamma(\alpha)}-\|\nu\|_{E} \frac{1+\alpha+\Gamma(\alpha)}{\Gamma(\alpha)} \int_{0}^{\infty} q(t) \theta(t) \mathrm{d} t\right] \\
& \geq \frac{\Gamma(\alpha)+(1 / 2)}{\Gamma(\alpha)+1}\|\nu\|_{E}\left[\delta \frac{1+\Gamma(\alpha)}{\Gamma(\alpha)}-\frac{1+\alpha+\Gamma(\alpha)}{\Gamma(\alpha)} \int_{0}^{\infty} q(t) \theta(t) \mathrm{d} t\right] \\
& =\|\nu\|_{E} .
\end{aligned}
$$

Then, we conclude that

$$
\|\nu\|_{E} \leq \frac{(\Theta \nu)\left(\tau_{0}\right)}{1+\tau_{0}^{\alpha-1}} \leq(\Theta \nu)\left(\tau_{0}\right) \leq\|\Theta \nu\|_{H} \leq \sigma\left(\nu_{0}\right)\|\Theta \nu\|_{H} .
$$

Step 8: here, we need to explain that $(P+J Q N)_{\mu}\left(\partial \Omega_{2}\right) \subset C$ is held, i.e., $\forall v \in \partial \Omega_{2}$, and we have $P+J Q N(|\nu(\tau)|) \in C$ :

$$
\begin{aligned}
P+J Q N(|\nu(\tau)|) & =\frac{\tau^{\alpha-1}}{\kappa(\tau)}\left[\frac{1}{\Gamma(\alpha)} \int_{0}^{\infty} e^{-t} \kappa(t)|\nu(t)| \mathrm{d} t+\int_{0}^{\infty} q(t) \beta(t,|\nu(t)|) \mathrm{d} t\right] \\
& \geq \frac{\tau^{\alpha-1}}{\kappa(\tau)}\left[\int_{0}^{\infty}\left(e^{-t}\left(1+t^{\alpha-1}\right)-q(t) \theta(t)\right) \frac{|\nu(t)|}{\left(1+t^{\alpha-1}\right)} \mathrm{d} t\right] \\
& \geq 0 .
\end{aligned}
$$

That makes $(P+J Q N)_{\mu}\left(\partial \Omega_{2}\right) \subset C$.

Step 9: by this step, $\Theta_{\mu}\left(\overline{\Omega_{2}} / \Omega_{1}\right) \subset C$ will be satisfied. For that take $v \in \overline{\Omega_{2}} / \Omega_{1}$. It follows that $R_{1} \leq|v(t)| \leq R_{2}$. Now, use $\left(\mathscr{H}_{4}(79)\right)$, we obtain 


$$
\Theta(|\nu(t)|) \geq \frac{\tau^{\alpha-1}}{\Gamma(\alpha) \kappa(\tau)}\left[\int_{0}^{\infty}\left(e^{-t}\left(1+t^{\alpha-1}\right)-(\Gamma(\alpha)+\alpha+1) q(t) \theta(t)\right) \frac{|\nu(t)|}{\left(1+t^{\alpha-1}\right)} \mathrm{d} t\right]
$$

$\geq 0$,

which means that $\Theta_{\mu}\left(\overline{\Omega_{2}} / \Omega_{1}\right) \subset C$ is hold.

Step 10: this step proves that $\nu \notin(P+J Q N)_{\mu} \nu$, $\forall \nu \in \operatorname{Ker} L \cap \partial \Omega_{2} \quad$ will be true. Consider $v \in \operatorname{Ker} L \cap \partial \Omega_{2}$, then $|b|=R_{2}$. Moreover,

$$
\begin{aligned}
P+J Q N\left(|b| \frac{\tau^{\alpha-1}}{\kappa(\tau)}\right) & =\frac{\tau^{\alpha-1}}{\kappa(\tau)}\left[\frac{1}{\Gamma(\alpha)} \int_{0}^{\infty} e^{-t} \kappa(t)|b| \frac{t^{\alpha-1}}{\kappa(t)} \mathrm{d} t+\int_{0}^{\infty} q(t) \beta\left(t,|b| \frac{t^{\alpha-1}}{\kappa(t)} \mathrm{d} t\right]\right. \\
& \leq \frac{\tau^{\alpha-1}}{\kappa(\tau)}\left[R_{2}-R_{2} \int_{0}^{\infty} q(t) \eta_{1}(t) \frac{t^{\alpha-1}}{\left(1+t^{\alpha-1}\right) \kappa(t)} \mathrm{d} t+\int_{0}^{\infty} q(t) \eta_{2}(t) \mathrm{d} t\right] \\
& \leq R_{2} .
\end{aligned}
$$

So, $v \notin(P+J Q N)_{\mu} \nu, v \in \operatorname{Ker} L \cap \partial \Omega_{2}$ is true.

Hence, problem (39), the steps (Step 1-Step 10), and (O'Regan and Zima, Theorem 1) together show that our problem (1)-(3) can attain a positive solution at resonance in the set $C \cap\left(\overline{\Omega_{2}} / \Omega_{1}\right)$.

\section{An Example}

Example 1. Taking problems (1)-(3) with respect to

$$
\begin{aligned}
\alpha & =\frac{5}{2}, \\
\kappa(\tau) & =2, \\
A(\tau, \nu(\tau)) & =\left[-\frac{1}{30} e^{-\tau} \nu(\tau)+\frac{1}{\lambda^{2}\left(1+\tau^{3 / 2}\right)\left(1+\tau^{2}\right)}\right]_{\lambda=1}^{m}, \quad \lambda \in \mathbb{N} .
\end{aligned}
$$

First, if $a(\tau, \nu(\tau)) \in A(\tau, \nu(\tau))$, we obtain

$$
\begin{aligned}
a(\tau, \nu(\tau)) & =-\frac{1}{30} e^{-\tau} \nu(\tau)+\frac{1}{\lambda^{2}\left(1+\tau^{3 / 2}\right)\left(1+\tau^{2}\right)} \\
& =\frac{1}{\left(1+\tau^{3 / 2}\right)}\left[-\frac{1}{30} e^{-\tau}\left(1+\tau^{3 / 2}\right)^{2} \frac{\nu(\tau)}{\left(1+\tau^{3 / 2}\right)}+\frac{1}{\lambda^{2}\left(1+\tau^{2}\right)}\right]
\end{aligned}
$$

From that we will take

$$
\begin{aligned}
q(\tau)= & \frac{1}{\left(1+\tau^{3 / 2}\right)}, \\
q_{0}= & 1, \\
B(\tau, \nu(\tau))= & {\left[-\frac{1}{30} e^{-\tau}\left(1+\tau^{3 / 2}\right)^{2} \frac{\nu(\tau)}{\left(1+\tau^{3 / 2}\right)}+\frac{1}{\lambda^{2}\left(1+\tau^{2}\right)}\right]_{\lambda=1}^{m}, } \\
& \lambda \in \mathbb{N}, \\
\beta(\tau, \nu(\tau))= & -\frac{1}{30} e^{-\tau}\left(1+\tau^{3 / 2}\right)^{2} \frac{\nu(\tau)}{\left(1+\tau^{3 / 2}\right)}+\frac{1}{\lambda^{2}\left(1+\tau^{2}\right)} .
\end{aligned}
$$

And then,

$$
\begin{aligned}
-\frac{1}{30} e^{-\tau}\left(1+\tau^{3 / 2}\right)^{2} \frac{\nu(\tau)}{\left(1+\tau^{3 / 2}\right)} & \leq \beta(\tau, \nu(\tau)) \\
& \leq-\frac{1}{30} e^{-\tau}\left(1+\tau^{3 / 2}\right)^{2} \frac{\nu(\tau)}{\left(1+\tau^{3 / 2}\right)}+\frac{1}{\left(1+\tau^{2}\right)}, \\
& \Longrightarrow \theta(\tau)=\eta_{1}(\tau) \\
\eta_{2}(\tau) & =\frac{1}{\left(1+\tau^{2}\right)} .
\end{aligned}
$$

Also,

$$
\begin{gathered}
\beta(\tau, \nu(\tau)) \leq|\beta(\tau, \nu(\tau))| \\
\leq \eta_{1}(\tau) \frac{\nu(\tau)}{\left(1+\tau^{3 / 2}\right)}+\eta_{2}(\tau) \\
\Longrightarrow-|\beta(\tau, \nu(\tau))| \mid \geq-\eta_{1}(\tau) \frac{\nu(\tau)}{\left(1+\tau^{3 / 2}\right)}-\eta_{2}(\tau) \\
\Longrightarrow \beta(\tau, \nu(\tau)) \leq-2|\beta(\tau, \nu(\tau))|+\eta_{1}(\tau) \frac{\nu(\tau)}{\left(1+\tau^{3 / 2}\right)}+3 \eta_{2}(\tau) \\
\Longrightarrow \gamma_{1}(\tau)=\gamma_{0}=2, \gamma_{2}(\tau)=\eta_{1}(\tau), \gamma_{3}(\tau)=3 \eta_{2}(\tau) .
\end{gathered}
$$

So, $\left(\mathscr{H}_{2}\right)$ is held.

To explore $\left(\mathscr{H}_{3}\right)$, we need to compute the values hereinafter: 


$$
\begin{aligned}
\Gamma\left(\frac{5}{2}\right) & =\frac{3}{4} \sqrt{\pi}, \\
\Gamma\left(\frac{5}{2}\right)+\frac{7}{2} \simeq 4.829 & <30, \\
2\left(\Gamma\left(\frac{5}{2}\right)+\frac{1}{2}\right) \simeq 3.659 & <30, \\
2\left(\Gamma\left(\frac{5}{2}\right)+\frac{1}{2}\right)\left(\Gamma\left(\frac{5}{2}\right)+\frac{7}{2}\right) \simeq 17.66901 & <30 .
\end{aligned}
$$

Now, we can illustrate that $\left(\mathscr{H}_{3}\right)$ is useful as follows:

$$
\begin{aligned}
q(\tau) \theta(\tau) & =\frac{1}{30} e^{-\tau}\left(1+\tau^{3 / 2}\right) \\
& \Longrightarrow e^{\tau} q(\tau) \theta(\tau)=\frac{1}{30}\left(1+\tau^{3 / 2}\right) \\
& <\frac{1+\tau^{3 / 2}}{\Gamma(5 / 2)+(7 / 2)}, \\
\int_{0}^{\infty} q(\tau) \theta(\tau) \mathrm{d} \tau & =\frac{1}{30}(\Gamma(5 / 2)+1) \\
& <\frac{\Gamma(5 / 2)+1}{2(\Gamma(5 / 2)+(1 / 2))(\Gamma(5 / 2)+(7 / 2))}, \\
\delta & \simeq 0.887655<1 .
\end{aligned}
$$

At the last, by calculating the upcoming values, we see that $\left(\mathscr{H}_{4}\right)$ is verified:

$$
\begin{aligned}
\eta_{0} & =\frac{3 \sqrt{\pi}}{4(60)}=\frac{3 \sqrt{\pi}}{240}, \\
\frac{q_{0}}{\eta_{0}} & =\frac{240}{3 \sqrt{\pi}}, \\
q_{0} & =1, \\
\gamma_{0} & =2, \\
\Gamma_{0} & =61, \\
\frac{q_{0} \Gamma_{0}}{\Gamma(5 / 2)} & =\frac{244}{3 \sqrt{\pi}}, \\
\frac{6 q_{0}}{\gamma_{0} \Gamma(5 / 2)} & =\frac{3}{3 \sqrt{\pi}}, \\
\int_{0}^{\infty} \frac{\pi}{\eta_{2}(t) \mathrm{d} t} & =\frac{\pi}{2}, \\
R_{0} & =\frac{\pi}{2}\left(\frac{244}{3 \sqrt{\pi}}\right)=\frac{122 \sqrt{\pi}}{3} .
\end{aligned}
$$

By adopted upshots and Theorem 2, the presented problem seems to be solvable.

\section{Conclusion}

Academically, we analyzed the potentialities of having a positive solution in unbounded domain (on the half line) for the presumed inclusion problems (1)-(3). As we see, getting the positive solution at resonance was contingent with using O'Regan and Zima, Theorem 1. In the way to see the importance of the new upshots, we spelled out by example. We picked out these ramifications, and fortunately we can verify them for the sake of finding some extent applications in the other scientific fields.

\section{Data Availability}

The data used to support the findings of this study are available from the corresponding author upon request.

\section{Conflicts of Interest}

The authors declare that they have no conflicts of interest.

\section{Acknowledgments}

This project was funded by the Deanship of Scientific Research (DSR), King Abdulaziz University, Jeddah, under Grant no. D-028-130-1438. The authors, therefore, gratefully acknowledge DSR for their technical and financial support.

\section{References}

[1] A. Kilbas, H. M. Sirvastava, and J. J. Trujilo, Theory and Applications of Fractional Differential Equation, Vol. 204, Elsevier Science, Amsterdam, Netherlands, 2006.

[2] G. V. Smirnov, "Introduction to the theory of differential inclusions," Graduate Studies in Mathematics, vol. 41, 2002.

[3] J. Santanilla, "Some coincidence theorems in wedges, cones, and convex sets," Journal of Mathematical Analysis and Applications, vol. 105, no. 2, pp. 357-371, 1985.

[4] A. Salem and M. Alnegga, "Fractional Langevin equations with multi-point and non-local integral boundary conditions," Cogent Mathematics \& Statistics, vol. 7, no. 1, Article ID 1758361, 2020.

[5] A. Salem and B. Aghamdi, "Multi-strip and multi-point boundary conditions for fractional Langevin equation," Fractal and Fractional, vol. 4, no. 2, pp. 1-13, 2020.

[6] A. Salem, F. Alzahrani, and M. Alnegga, "Coupled system of non-linear fractional Langevin equations with multi-point and nonlocal integral boundary conditions," Mathematical Problems in Engineering, vol. 2020, Article ID 7345658, 15 pages, 2020.

[7] A. Salem, F. Alzahrani, and B. Alghamdi, "Langevin equation involving two fractional orders with three-point boundary conditions," Differential and Integral Equations, vol. 33, no. 34, pp. 163-180, 2020.

[8] A. Salem, F. Alzahrani, and L. Almaghamsi, "Fractional Langevin equation with nonlocal integral boundary condition," Mathematics, vol. 7, no. 5, pp. 1-10, 2019.

[9] A. Salem and B. Aghamdi, "Multi-point and anti-periodic conditions for generalized Langevin equation with two fractional orders," Fractal and Fractional, vol. 3, no. 4, pp. 1-14, 2019.

[10] A. Salem and A. Al-Dosari, "Existence results of solution for fractional Sturm-Liouville inclusion involving composition 
with multi-maps," Journal of Taibah University for Science, vol. 14, no. 1, pp. 721-733, 2020.

[11] Y. Chen and X. Tang, "Positive solution of fractional differential equation at resonance on the half line," Boundary Value Problems, vol. 64, pp. 1-13, 2012.

[12] W. Jiang and C. Yang, "The existence of positive solutions for multi-point boundary value problem at resonance on the halfline," Boundary Value Problems, vol. 13, pp. 1-12, 2016.

[13] Y. Chen, X. Tang, and X. He, "Positive solutions of fractional differential inclusions at resonance," Mediterranean Journal of Mathematics, vol. 10, no. 3, pp. 1207-1220, 2013.

[14] L. Hu, "On the existence of positive solutions for fractional differential inclusion at resonance," SpringerPlus, vol. 5, no. 957, pp. 1-12, 2016.

[15] Y. Wang and L. Liu, "Positive solutions for a class of fractional 3-point boundary value problems at resonance," Advances in Difference Equations, vol. 2017, pp. 1-13, 2017.

[16] G. Haller, Chaos Near Resonance, Volume 138 of Applied Mathematical Sciences, Springer-Verlag, New York, NY, USA, 1999.

[17] V. Lakshmikantham, S. Leela, and J. V. Devi, Theory of Fractional Dynamic Systems, Cambridge Scientific Publishers Ltd, Cambridge, MA, USA, 2009.

[18] S. K. Miller and B. Ross, An Introduction to the Fractional Calculus and Fractional Differential Equation, Wiley, Hoboken, NJ, USA, 1993.

[19] J.-P. Aubin and A. Cellina, Differential Inclusion: Set-Valued Maps and Viability Theory, Springer-Verlag, Berlin, Heidelberg, Germany, 1984.

[20] J.-P. Aubin and H. Frankowska, Set-valued Analysis, Birkhäuser, Boston, MA, USA, 2009.

[21] K. Deimling, Multi-valued Differential Equations, De Gruyter Series in Nonlinear Analysis and Applications, Vol. 1, Walter de Gruyter, Berlin, NY, USA, 1992.

[22] J. Mawhin, "Equivalence theorems for nonlinear operator equations and coincidence degree theory for some mappings in locally convex topological vector spaces," Journal of Differential Equations, vol. 12, no. 3, pp. 610-636, 1972.

[23] D. O'Regan and M. Zima, "Laggett-Williams theorems for coincidence of multi-valued operators," Nonlinear Analysis: Theory, Methods \& Applications, vol. 68, no. 10, pp. 28792888, 2008.

[24] W. V. Petryshyn, "On the solvability of $x \in T x+\lambda F x$ in quasinormal cones with a $T$ and $F k$-set contractive," Nonlinear Analysis: Theory, Methods \& Applications, vol. 5, pp. 589-591, 1981.

[25] X. Zaho and W. Ge, "Unbounded solutions for a fractional boundary value problems on the infinite interval," Acta Applicandae Mathematicae, vol. 109, pp. 459-505, 2010.

[26] R. P. Agrwal and D. O’Regan, Infinite Interval Problems for Differential and Integral Equations, Springer Science \& Business Media, Berlin, Germany, 2012. 